\title{
ESTÍMULOS TRIBUTARIOS Y HOTELERÍA EN COLOMBIA
}

JOSÉ MANUEL LEGUIZAMÓN TIUSABÁ*

\begin{abstract}
RESUMEN
Los últimos gobiernos han propiciado la inversión y la cualificación de la gestión en el sector turismo mediante estímulos tributarios. Particularmente el sector alojamiento ha tenido un tratamiento diferenciado en el marco de la tributación directa e indirecta. Un claro ejemplo de este tipo de estímulos tributarios lo constituye la promulgación de la Ley 788 de 2002 que originó un crecimiento inédito de la oferta habitacional hotelera en Colombia. Sin embargo, algunos expertos en materia fiscal y turística consideran que este tipo de incentivos es discriminatorio, por concentrarse en unos pocos beneficiarios. También se argumenta que el Estado deja de recaudar recursos importantes que, de recibirse, se podrían invertir en la misma infraestructura que requiere la actividad turística para su desarrollo. Este artículo mide y analiza los impactos de estas disposiciones fiscales y tributarias en la operación y la gestión del sector alojamiento de Colombia.
\end{abstract}

Palabras clave: Colombia, turismo, política tributaria, hotelería

Clasificaciones JEL: C01, C13, C22, C35, C53, E52

* El autor es director de Infoconsultoría, y docente de cátedra de la Universidad Externado de Colombia y de la Universidad Tecnológica de Bolívar. Correo electrónico: manuel.leguizamon2@gmail.com. Recibido: 6 de marzo de 2019; aceptado: 8 de junio de 2019.

Economía \& Región, Vol. 13, No. 1, (Cartagena, junio 2019), pp. 177-198. 


\section{ABSTRACT \\ Effect of tax incentives on the Colombian hotel sector}

Recent governments have introduced tax incentives to promote investment and increase the quality of tourist services. In particular, the lodging sector has received differential treatment in direct and indirect taxes. A clear example of this type of tax stimulus is Law 788 from 2002, which resulted in an unprecedented increase in hotel capacity in Colombia. However, some fiscal and tourism experts have viewed this type of incentive as discriminatory, because it benefits the few. They also argue that the government fails to collect significant tax revenues that could be invested in the same infrastructure required by the tourist sector for its development. The purpose of this paper is to measure and analyze the impacts of these fiscal and tax policies on the operation and management of the lodging sector of Colombia.

Key words: Colombia, tourism, tax policy, hotels

JEL Classifications: C01, C13, C22, C35, C53, E52

\section{INTRODUCCIÓN}

El sector hotelero colombiano recibió beneficios tributarios en los tres últimos lustros. Los estímulos se fundamentaron en la promulgación y el desarrollo de políticas tributarias, como la Ley 788 de 2002 cuyo cuerpo normativo estableció beneficios tributarios para el sector de alojamiento del país.

Estos beneficios buscaron estimular a la inversión, generar empleo, ayudar a la formalización empresarial, mejorar la gestión, e incentivar la inversión extranjera. Así mismo, estos beneficios pretendieron el efecto indirecto de potenciar a Colombia como destino turístico internacional.

Desde 2002, cuando se promulgó la mencionada Ley 788, creció la planta hotelera, se dio la construcción o remodelación inmobiliaria destinada a servicios de alojamiento, aparecieron cadenas hoteleras internacionales y, probablemente, se cualificación la gestión y los servicios en el sector. Algunos operadores hoteleros, sin embargo, consideran que estos beneficios se pudieron haber diluido por reformas posteriores que impactaron la operación y la rentabilidad de la 
prestación del servicio (UT Infoconsultoria-Yanhaas, 2016, p. 240). Por tanto, surgió el interés de analizar cómo estos beneficios tributarios influyeron en el sector alojamiento.

UT Infoconsultoria-Yanhaas (2016) examinó cómo las últimas reformas tributarias y los estímulos tributarios decretados influyeron en la hotelería colombiana. UT Infoconsultoria-Yanhaas (2016) fue un análisis comparativo de los casos de España, México y Argentina. Usó una muestra del sector colombiano de la hotelería y fuentes secundarias institucionales (i.e. DIAN, DANE, entre otras). Otras instituciones del sector turístico, como los gremios y la academia, han estudiado esta pregunta de investigación.

Este artículo corresponde a un análisis ampliado de los principales resultados de estos estudios, particularmente de UT Infoconsultoria-Yanhaas (2016), al aprovechamiento de los registros o bases de datos construidos, a la convalidación actualizada de los resultados y al balance de los impactos de los estímulos tributarios que se han derivado tanto para el Estado en materia de ingresos fiscales como para el sector alojamiento del país, en especial en aspectos relacionados con la operación y la gestión

\section{MÉTODO}

Este artículo corresponde a una investigación aplicada sobre normas y disposiciones de tipo tributario que tuvieron un especial impacto jurídico y económico en la gestión y la operación de la hotelería colombiana. Se estudiaron las reformas tributarias de últimos quince años cuyo cuerpo normativo tuvo incidencia en el sector turismo, en especial el hotelero. En este sentido la metodología se basó en técnicas desarrolladas con los datos de investigaciones referentes para este estudio, más otros obtenidos de fuentes secundarias para actualizar los registros.

Fue así como, de acuerdo con la base de datos obtenida de UT Infoconsultoria-Yanhaas (2016), el cual tuvo cobertura nacional, se realizó un análisis econométrico y estadístico a partir del modelamiento de datos provenientes de la muestra probabilística desarrollada con los establecimientos hoteleros y con el recurso de las bases de datos institucionales, como las de la DIAN, DANE y Cotelco.

La muestra probabilística de la cual se obtuvieron los datos primarios de este estudio correspondió a una de tipo probabilística, estratificada con selección Proporcional al Tamaño -PPT. El marco de muestreo lo constituyó el Registro 
Nacional de Turismo que contiene los establecimientos denominados con la categoría de "hotel", los cuales fueron las unidades de muestreo. Cubrió las doce ciudades más importantes para el turismo nacional, según la oferta de habitaciones hoteleras.

Los estratos se conformaron según el rango de habitaciones de estos establecimientos:

\section{CUADRO 1}

Distribución de la muestra según estratos

\begin{tabular}{|c|c|c|}
\hline Estratos & Rangos de Hab. & Número de hoteles \\
\hline 1 & 40 o menos & 2.209 \\
\hline 2 & 41 a 99 & 411 \\
\hline 3 & 100 a 149 & 55 \\
\hline 4 & 150 o más & 57 \\
\hline
\end{tabular}

Fuente: UT Infoconsultoria-Yanhaas (2016).

De manera que estos cuatro estratos corresponden al criterio de pequeña, media baja y media alta y gran hotelería. Basados en este diseño los resultados resultan ponderados y representativos según tamaño de los hoteles. La información obtenida de estas fuentes primarias fue complementada con los registros institucionales del DANE, la DIAN y Cotelco.

De acuerdo con en esta base de datos ensamblada y para los propósitos de este artículo se emplearon métodos y técnicas econométricas y estadísticas para el modelamiento de los datos. Se diseñaron modelos de regresión múltiple jerárquica y stepwise, ANOVA, pruebas de significancia y pruebas de correlación de forma tal que se buscaron aquellas variables que resultaran más significativas en la explicación del impacto en la gestión y la operación. Estas variables fueron: Ingresos, IVA, P\&G, IPC, Empleo y Ocupación.

A continuación, se realizó un análisis multivariado con el método de Componentes Principales el cual "permite pasar de un conjunto de variables correlacionadas entre sí a otro en el que las dimensiones (las componentes principales) están incorrelacionadas [sic] y ordenadas en función de la información que llevan incorporada" (Uriel y Aldás, 2005, p. 393). Esta técnica permitió reconocer los 
factores para caracterizar las variables que tuvieron el mayor impacto en atención a las diferentes reformas tributarias en el periodo de estudio considerado.

En este sentido los cambios en impuestos directos e indirectos, como el IVA, fueron considerados para medir el impacto en factores determinantes del negocio hotelero, como: ocupación, tarifas, utilidad operacional, ingresos por alojamiento y empleo, entre los más importantes.

\section{RESULTADOS Y DISCUSIÓN}

El sistema impositivo colombiano se ha ajustado casi cada dos años en los últimos veinticinco a través de diferentes reformas tributarias. A los inversionistas y a los gestores les generaron incertidumbre estos ajustes, como es natural. El sector alojamiento no ha sido la excepción a estos cambios.

Sin embargo, es justo destacar que la hotelería en casos significativos tuvo favorecimientos especiales como quiera que algunas reformas buscaran dinamizar e impulsar esta actividad turística mediante el otorgamiento de beneficios fiscales. Estos se generaron en el marco de un conflicto armado en Colombia del cual el sector turístico era uno de los más damnificados.

Como se sabe, el turismo requiere para su desarrollo de unas condiciones especiales en términos de supra estructura, infraestructura, atractivos, facilitadores, conectividad y seguridad. Como se indica en Leguizamón (2017, p. 253): "el viaje turístico es generador de una demanda de bienes y servicios turísticos y no turísticos que son diseñados, producidos, facilitados y consumidos en un entorno económico por el turista. Se destaca en esta perspectiva la estimación del consumo de visitantes en la relación con la oferta de bienes y servicios de las industrias turísticas, esencialmente, y las conexas".

Por tanto, estos estímulos tributarios directos e indirectos para este sector deben también leerse como una forma de apoyar una actividad económica generadora de empleo e inversión que en tiempos de conflicto armado resulta muy afectada. Desde la perspectiva de los impuestos del orden nacional estos impactan la renta de las sociedades jurídicas que prestan los servicios de alojamiento, ya que estos se determinan de acuerdo con sus ingresos operacionales y las particularidades de este sector.

De otra parte, los estímulos para estos operadores y prestadores del sector de la hotelería han estado sustentados en la disminución de la base gravable o la 
diferenciación de algunos impuestos como el Impuesto al Valor Agregado (IVA) como más adelante se describe.

No menos significativa es la posibilidad de deducir fiscalmente algunos gastos de ocurrencia frecuente en la operación hotelera. Por tanto, el análisis del impacto tributario en actividades como los servicios de alojamiento temporal reviste gran trascendencia para establecer la viabilidad y el espacio de atracción que se propicia para el inversionista y el operador.

Retomando el contexto de las reformas tributarias de los últimos veinticinco años y significando lo correspondiente a la actividad turística, es importante empezar por referir la Ley 223 de $1995,{ }^{1}$ que tuvo como fundamento el aumento de la tarifa del IVA al 16\%, la regulación del mecanismo de retención en la fuente y otras modificaciones relacionadas con la tributación directa. Para este momento la tarifa del IVA para el sector hotelero era del 10\%. Entonces puede considerarse que este es un primer evento de las reformas tributarias al sector.

Solamente tres años después, la Ley 488 de $1998^{2}$ modificó el tratamiento tributario de los servicios de alojamiento al incluirlos dentro del listado de servicios excluidos, es decir este impuesto no se descuenta en el manejo contable, ya que no se genera. Este tratamiento tributario no necesariamente es favorable, pues como no se puede descontar en las fases secuenciales de la producción del servicio termina trasladándose al consumidor final.

$\mathrm{Al}$ respecto, expertos tributaritos coinciden en considerar que, a pesar de la interpretación popular, la exclusión del pago del IVA no conlleva necesariamente a una reducción de las tarifas, como bien lo indica UT Infoconsultoria-Yanhaas (2016, p. 100): "No obstante, la sensación que genera en el imaginario colectivo es que esta exclusión del IVA 'abarata' el servicio, aspecto que en términos económicos y financieros no es exacto, por lo cual la tendencia en los mejores modelos de tributación es establecer el beneficio bajo la forma de exención del servicio, el cual como ya lo hemos explicado sí permite el descuento del IVA pagado". ${ }^{3}$

Los diferentes destinos turísticos buscan mantener y aumentar su participación en el mercado de los viajes. Esta participación se mejora mediante el desarrollo de

${ }^{1}$ Congreso de Colombia. (1995). Ley 223 de 1995. Recuperado de http://www.secretariasenado.gov.co/ senado/basedoc/ley_0223_1995.html

${ }^{2}$ Congreso de Colombia. (1998). Ley 488 de 1998. Recuperado de http://www.secretariasenado.gov.co/ senado/basedoc/ley_0488_1998.html

${ }^{3}$ Concepto DiAn No. 09115 del 26 de marzo de 2015. 
estrategias alternativas. Unas corresponden a temas de mercadeo, otras de publicidad y posicionamiento de marca; también las de diversificación de productos y servicios turísticos, entre otras estrategias. En este propósito los estímulos tributarios a los turistas residentes en el exterior, por su evidente eficacia, son de uso común en los destinos turísticos líderes.

En esta lógica la Ley 633 del $2000^{4}$ corresponde a un estímulo directo al turismo internacional que visita Colombia. En efecto, su cuerpo normativo estableció la exención para los servicios turísticos prestados en nuestro territorio a visitantes residentes en el exterior. Estos servicios debían ser ofrecidos y prestados por operadores turísticos con Registro Nacional de Turismo (RNT) vigente.

En efecto, el Artículo 58. Servicios Turísticos, estableció:

“e) También son exentos del impuesto sobre las ventas los servicios que sean prestados en el país en desarrollo de un contrato escrito y se utilicen exclusivamente en el exterior, por empresas o personas sin negocios o actividades en Colombia, de acuerdo con los requisitos que señale el reglamento. Recibirán el mismo tratamiento los servicios turísticos prestados a residentes en el exterior que sean utilizados en territorio colombiano, originados en paquetes vendidos en el exterior y vendidos por agencias operadoras inscritas en el Registro Nacional de Turismo, según lo establecido en la Ley 300 de 1996, y siempre y cuando se efectúe el respectivo reintegro cambiario". 5

Pero lo que puede considerarse como un punto de inflexión en materia de estímulos tributarios para el sector turístico colombiano y en especial para la hotelería, lo constituye la promulgación y la reglamentación de la Ley 788 de $2002 .{ }^{6}$ Esta ley creó una serie de incentivos de tipo fiscal y tributario para estimular la inversión en la construcción o la remodelación de establecimientos con destino a la prestación de servicios de alojamiento temporal. Los pilares de esta ley estaban fundamentados en la exención del impuesto de renta por treinta años a los hoteles que se construyeran o remodelaran entre 2002 y 2017 . La Ley 788 de 2002 en su artículo 18 decretó lo siguiente:

\footnotetext{
${ }^{4}$ Congreso de Colombia. (2000). Ley 633 del 2000. Recuperado de http://www.mineducacion.gov.co/ 1621/articles-270478_recurso_1.pdf

${ }^{5}$ Recuperado de www.secretariasenado.gov.co/senado/basedoc/

${ }^{6}$ Ley 788 de 2002. Diario oficial No. 45.046, 27 de diciembre de 2002
} 
"Numeral 3: Servicios hoteleros prestados en nuevos hoteles que se construyan dentro de los quince años siguientes a partir de la vigencia de la presente ley, por un término de treinta años.

Numeral 4: Servicios hoteleros prestados en hoteles que se remodelen y/o amplíen dentro de los quince años siguientes a la vigencia de la presente ley, por un término de treinta años. La exención prevista en este numeral corresponderá a la proporción que represente el valor de la remodelación y/o ampliación en el costo fiscal del inmueble remodelado y/o ampliado, para lo cual se requiere aprobación previa del proyecto por parte de la curaduría urbana y a la alcaldía municipal, del domicilio del inmueble remodelado y/o ampliado. (Ley 788 de 2002, Art. 18)”

Esta Ley se reglamentó mediante el decreto 2755 del 2003 el cual estableció que los nuevos hoteles o los remodelados a partir del 1 de enero de 2003 eran beneficiados con la exención del Impuesto a la Renta por treinta y quince años, respectivamente.

"Artículo $4^{\circ}$. Renta exenta en servicios hoteleros prestados en nuevos hoteles. Modificado por el art. 1, Decreto Nacional 920 de 2009. Las rentas provenientes de servicios hoteleros prestados en nuevos hoteles construidos a partir del $1^{\circ}$ de enero de 2003, obtenidas por el establecimiento hotelero o por el operador según el caso, están exentas del impuesto sobre la renta por un término de 30 años, contados a partir del año gravable 2003.

Para tal efecto se consideran nuevos hoteles aquellos cuya construcción se inicie y termine dentro de los 15 años siguientes, contados a partir del $1^{\circ}$ de enero de 2003" (Decreto 2755 de 2003, Artículo 4).

Este Decreto estableció, además, que únicamente los servicios provenientes de hoteles eran los beneficiados con el incentivo fiscal:

"Parágrafo. Los ingresos provenientes de los servicios de moteles, residencias, y establecimientos similares no se encuentran amparados por la exención prevista en este artículo (Decreto 2755 de 2003, Artículo 4)”.

En cuanto a los establecimientos remodelados la exención guardó proporcionalidad con el monto de lo invertido en la remodelación: 
“Artículo 6. La exención corresponderá a la proporción que represente el valor de la remodelación y/o ampliación en el costo fiscal del inmueble remodelado y/o ampliado. (Decreto 2755 de 2003, Artículo 6)"

A partir de esta reglamentación fue necesario precisar los beneficios de estos estímulos pues se generó confusión en el sentido de quiénes serían los beneficiados (inversionistas, constructores u operadores turísticos) y su vigencia. El Decreto 920 de 2009 aclaró estos aspectos de manera que los beneficios se establecieron a partir del año gravable de inicio de operaciones.

En consecuencia, la Ley 788 del 2002 buscó incentivar la oferta, en este caso habitaciones hoteleras; reguló la renta exenta por prestación de servicios de alojamiento. El resultado inmediato de estos beneficios representó el incremento de manera sostenida de la oferta habitacional. Este crecimiento, por encima de los promedios históricos, llevó sin embargo a niveles de sobreoferta en los principales destinos turísticos.

Antes de analizar cómo fueron los efectos en el sector alojamiento en atención a estos beneficios, es pertinente mencionar lo establecido en la Ley 1111 del 2006. En esta se reglamentó la exención del IVA únicamente para servicios turísticos prestados y consumidos en el territorio colombiano por no residentes, y ofrecidos por prestadores con RNT vigente.

Otra reforma clave y que en opinión de algunos gestores fue la hostil para la gestión y la operación de la actividad turística, lo constituyó la Ley 1607 de 2002 ? Al respecto, dentro de lo más sobresaliente para el sector alojamiento está la regulación de la tarifa del IVA del 16\%. Y, otro pilar que trajo esta Ley fue la creación del Impuesto Nacional del Consumo del 8\% para los servicios prestados por los restaurantes. Este último ajuste afectó al sector en atención a que la prestación del servicio de expendio de alimentos y bebidas es preparada en restaurantes, así como la venta de bebidas alcohólicas para consumo dentro de bares. Esta nueva disposición acarreó sobresaltos a los gestores de este sector pues, como es bien conocido, muchos hoteles ofrecen el servicio de restaurante; y esta reglamentación no permitía descontar ningún tributo en la cadena de producción, como sí lo permite el IVA.

\footnotetext{
${ }^{7}$ Congreso de Colombia. (2012). Ley 1607 de 2012. Recuperado de http://www.secretariasenado.gov.co/ senado/basedoc/ley_1607_2012.html
} 
Así las cosas, a muchos establecimientos hoteleros se les generó dos impuestos simultáneamente, el del IVA del 16\% y el del Impuesto al Consumo del $8 \%$ en aspectos de tributación indirecta (uno deducible, el otro no). Esto les representó dificultades en la gestión estas a partir de estas nuevas disposiciones tributarias.

Posteriormente se promulgaron otras disposiciones tributarias como la reforma establecida en la Ley 1739 de $2014^{8}$, que creó, entre otras disposiciones, el Impuesto a la Riqueza, lo que indirectamente afectó a los inversionistas del sector hotelero, cuando estos tienen el carácter de personas naturales. Esta reforma generó una sobretasa al CREE que afectó a los hoteles.

En 2016 se produjo una nueva reforma denominada por el gobierno como estructural, la Ley $1819^{9}$ de ese año. El principal cambio al sistema tributario fue el aumento del IVA del 16\% al 19\% y la penalización a los evasores. Los servicios de alojamiento fueron también incluidos dentro de esta nueva tarifa.

En resumen, en estos últimos veinticinco años el sistema tributario colombiano ha tenido cerca de doce reformas. Por tanto, sería inalcanzable estudiar el impacto de cada una de ellas. Más bien el alcance de este artículo es centrarse en el análisis de los impactos más relevantes que trajeron aquellas reformas y que claramente tuvieron su influencia en la actividad turística, particularmente al sector alojamiento, actividad emblemática de la industria de los viajes.

La Ley 788 de 2002 marcó un nuevo derrotero en la industria turística en temas de desarrollo, gestión, operación y competitividad. Se hace referencia en particular a esta reforma porque indudablemente al sector turístico nacional le originó una dinámica de crecimiento inédita hasta ese momento; sin embargo, al Estado estos ajustes le generaron consecuencias fiscales y a los actores privados diferentes reacciones en temas de inversión y rendimientos.

En efecto para otros actores establecer una política de incentivos fiscales para un reducido grupo de inversionistas resulta discriminatorio y menoscaba la capacidad competitiva de la pequeña y micro empresa, como sostiene De la Santa (2017, p. 9): "las vacaciones del impuesto a la renta de 12 años era demasiado, argumentando que las vacaciones de impuesto sobre la renta para hoteles ya muy rentables solo sirven para enriquecer aún más a unos pocos selectos en lugar de mejorar el entorno general para el turismo".

\footnotetext{
${ }^{8}$ Recuperado de http://www.secretariasenado.gov.co/senado/basedoc/ley_1739_2014.htm

${ }_{9}^{9}$ Recuperado de: https://www.dian.gov.co/Prensa/HistoricoCentrales/Ley1819Diciembre2016.pdf
} 
Como se ha mencionado, la Ley 788 de 2002 tuvo como pilar fundamental estimular la inversión en el sector turístico colombiano en especial en infraestructura hotelera, uno de sus prestadores emblemáticos. En efecto se buscó incrementar la oferta habitacional y la cualificación de la infraestructura existente, en un entorno de apertura comercial.

La oferta de hoteles en Colombia presentó una tendencia de crecimiento sostenida durante el periodo de incidencia de esta reforma, como lo muestra el Gráfico 1. En términos de habitaciones este crecimiento representó un incremento del $369 \%$ en este mismo periodo (2005-2017). Se puede entonces inferir que esta reforma cumplió el propósito de incentivar la inversión en infraestructura hotelera.

La oferta hotelera colombiana creció entonces en este periodo gracias a las exenciones fiscales contempladas por la Ley 788 del 2002. Pero, este crecimiento originó una sobreoferta en los principales destinos turísticos, como Bogotá, Medellín y Cartagena.

Bogotá fue un destino turístico en el que la oferta habitacional en la década de 2003 a 2013 creció a un ritmo del 28\%, como se consigna en Debates Contemporáneos sobre el Turismo (2016, p. 28): "La oferta de establecimientos hoteleros de la ciudad se incrementó significativamente en la década de 2003 a 2013; para el

\section{GRÁFICO 1}

Colombia: Establecimientos hoteleros, 2005-2017

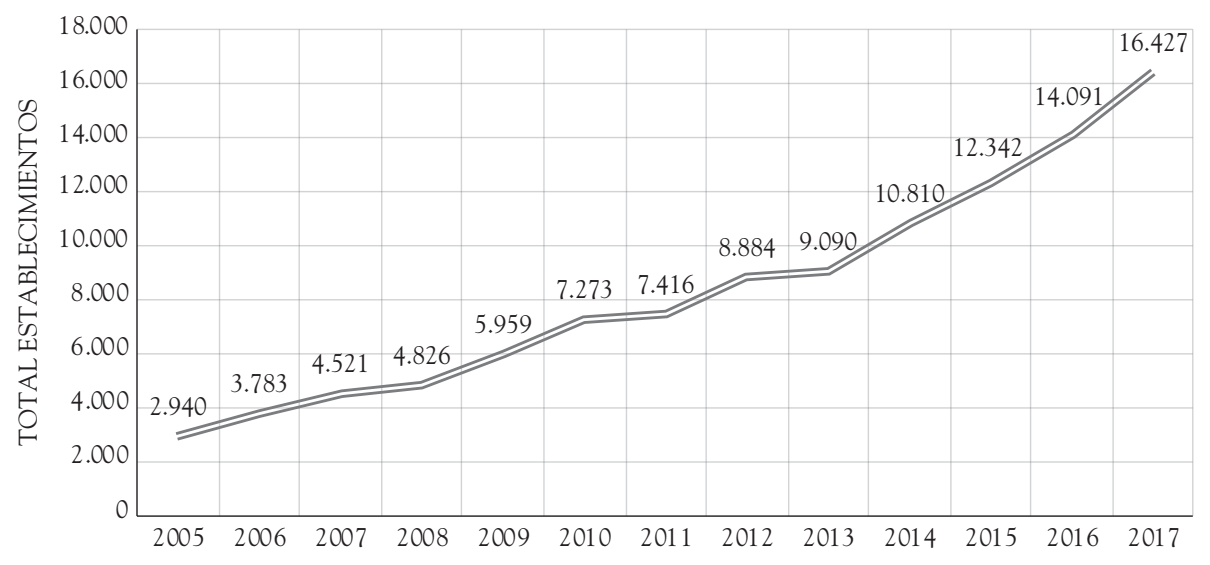

Fuente: Elaboración propia a partir de Citur, Mincit. 
2003 la ciudad contaba con cuatrocientos sesenta y dos (462) establecimientos, según datos de Cotelco. Para 2013, el total de establecimientos de la ciudad que fueron inscritos ante el Registro Nacional de Turismo como prestadores de servicios de alojamiento sumaban quinientos noventa y dos (592). Para un acumulado de 21.031 habitaciones y de 47.600 camas hoteleras".

Esto significa que la ciudad creció, en este periodo, a un ritmo de trece nuevos hoteles por año; es decir un promedio de uno nuevo por mes. Pero, mientras tanto, la demanda se mantuvo a una tasa estable (plana) y en algunos años de manera decreciente.

La demanda hotelera se mide por el numero de pernoctaciones. En el periodo de referencia, de crecimiento de la oferta habitacional, la demanda no crecio al mismo ritmo: "El número promedio mes de pernoctaciones en la serie estudiada comienza en el año 2003, con un estimado de 1.892.304; tuvo su mejor comportamiento en el año 2011 con una estimación mes de 2.629.992. Sin embargo, tuvieron una caída del 11\%, para el siguiente año." (Debates Contemporáneos sobre el Turismo et al, 2016, p. 35)

Este desbalance entre una oferta creciente y una demanda estable generó una sobreoferta de habitaciones hoteleras. La gestión de estos establecimientos se afectó. Los principales indicadores afectados fueron: ingresos operacionales, índices de ocupación, revenue par, empleo, tarifas, $P \& G$, entre otros.

Por su parte la inversión en el sector turismo se estudia en su contexto económico. Como se mencionó anteriormente, uno de los pilares de la Ley 788/2002 fue estimular la inversión especialmente en infraestructura hotelera. Para algunos entendidos el modelo económico que contextualiza esta lógica es la del neoliberalismo (UT Infoconsultoria-Yanhaas, 2016 p. 95).

Esta corriente económica se sustenta en la exclusión del Estado en atribuciones como la libertad de mercados, la apertura comercial, la economía; se atribuye al premio nobel de economía Milton Friedman (Friedman, 1962). Steger y Roy (2010), sostienen que esta doctrina se basa en los principios de desregulación de la economía, la liberalización del comercio y la industria, y la privatización de las empresas estatales.

Se observan rasgos comunes de la inversión en infraestructura hotelera con la Inversion Extranjera Directa (IED). Como dice la CEPAL (2006. p. 70) "la IED por si sola no resuelve los problemas relacionados con el desarrollo y el crecimiento económico, esta puede asumir un papel importante, en la medida que responda a los objetivos estratégicos de los países receptores". 
De manera que la IED comprende los condicionantes de las inversiones especialmente provenientes del capital internacional (competir en condiciones idénticas con las empresas locales, la garantía de un marco jurídico con procedimientos administrativos regulados y estables) los que guardan relación con esta política tributaria.

La reducción de impuestos a las empresas es otra característica coincidente con los estímulos tributarios otorgados para el sector de alojamiento. Su lógica es que los impuestos que deja de pagar el privado los convierta en infraestructura para generar inversión, consumo intermedio y empleo.

Esta reforma tributaria produjo resultados altamente significativos en materia de inversión para el desarrollo de la actividad turística. La inversión en infraestructura turística resultante de estos estímulos tributarios correspondió a $\$ 6$ billones entre los sectores público y privado, en los últimos 8 años. En infraestructura hotelera la inversión total fue de $\$ 17.541$ millones ("Turismo, Publicaciones Semana, Julio 2018”).

Estos resultados guardan coherencia con la política turística de los últimos gobiernos como es la de estimular la actividad mediante beneficios tributarios. Pero, como se advirtió antes, no son consensuados los beneficios de esta estrategia, pues se considera que es mejor recaudar el impuesto y usarlo para mejorar la infraestructura que requiere el mismo turismo para su desarrollo.

De otra parte, los establecimientos hoteleros tuvieron un impacto en su operación y su gestión como consecuencia del desarrollo de las últimas reformas. Especialmente se debe examinar lo generado a partir de la Ley 1607 de 2012, la cual, aunque retomó la exención de la tarifa del IVA, unificó todas las operaciones de los servicios de hotelería al 16\%. Es decir, se incrementó esta tarifa en seis puntos porcentuales, pues la tarifa que se traía era del $10 \%$ para estos mismos servicios.

El análisis de las repercusiones de este incremento del IVA es de interés manifiesto para esta industria. Se considera que el sector es muy sensible a un aumento en esta tarifa. Los indicadores financieros y operacionales reflejan estas afectaciones.

La investigación de estos efectos se basa en el análisis financiero. Autores como Scheel (2010) definen este análisis como la evaluación de los recursos desde su origen, la aplicación de estos y los resultados en términos de liquidez, rentabilidad y estabilidad financiera.

En este artículo se realiza un análisis de los efectos de las reformas tributarias en los establecimientos hoteleros a partir de las mediciones obtenidas en las principales variables que reflejan la gestión y la operación de estos. Estas variables fueron: Ingresos, IVA, P\&G, IPC, Empleo y Ocupación. 
Se usó la información disponible en UT Infoconsultoria-Yanhaas (2016), con registros que provienen de la muestra representativa de hoteles en Colombia más otros datos institucionales de la DIAN y el DANE para el periodo de estudio entre 2004 y 2014. Con esta información se realizó un análisis econométrico y estadístico.

Inicialmente se ajustó un modelo Arima. Los resultados fueron no significativos estadísticamente. Sin embargo, estos resultados facilitaron el diseño de modelos lineales, en los que se halló que la variable Ingresos resultó significativa para la explicación el modelo para P\&G y Empleo.

\section{CUADRO 2}

Resultados del Modelo Lineal (PEG, Empleo)

\begin{tabular}{|c|l|r|r|c|c|c|}
\hline \multirow{2}{*}{ Modelo } & \multicolumn{2}{|c|}{ Coeficientes no estandarizados } & $\begin{array}{c}\text { Coeficientes } \\
\text { estandarizados }\end{array}$ & \multirow{2}{*}{ t } & \multirow{2}{*}{ Sig. } \\
\cline { 3 - 6 } & \multicolumn{1}{|c|}{ B } & Error estándar & \multicolumn{1}{c|}{ Beta } & & \\
\hline \multirow{2}{*}{1} & (Constante) & 459.224 .921 & 86.476 .898 & & 5,31 & 0,000 \\
& P \& G & 9.121 & 0,442 & 0,99 & 20,62 & 0,000 \\
\hline \multirow{2}{*}{2} & (Constante) & 540.424 .028 & 66.304 .649 & & 8,15 & 0,000 \\
& P \& G & 9.318 & 319 & 1.011 & 29,22 & 0,000 \\
& EMPLEO & -5.120 .151 .891 & 1.618 .498 .217 & $-0,109$ & $-3,16$ & 0,013 \\
\hline
\end{tabular}

Fuente: Cálculos del autor.

La variable Ingresos fue definida como respuesta. Al ajustar el modelo se halló que el IVA resultó altamente significativa

\section{CUADRO 3}

Resultados del Modelo Lineal (IVA)

\begin{tabular}{|c|l|r|r|c|c|c|}
\hline \multirow{2}{*}{ Modelo } & \multicolumn{2}{|c|}{ Coeficientes no estandarizados } & $\begin{array}{c}\text { Coeficientes } \\
\text { estandarizados }\end{array}$ & \multirow{2}{*}{ t } & \multirow{2}{*}{ Sig. } \\
\cline { 3 - 5 } \multicolumn{2}{|c|}{} & \multicolumn{1}{|c|}{ B } & Error estándar & Beta & & \\
\hline \multirow{2}{*}{1} & (Constante) & -185.391 .927 & 122.375 .535 & 0,988 & $-1,52$ & 0,164 \\
& IVA & 18,42 & 0,944 & 19,51 & 0,000 \\
\hline
\end{tabular}

Fuente: Cálculos del autor. 
A continuación, se realizó un análisis multivariado con el método de Componentes Principales la cual es una técnica "que permite pasar de un conjunto de variables correlacionadas entre sí a otro en el que las dimensiones (las componentes principales) están incorrelacionadas y ordenadas en función de la información que llevan incorporada" (Uriel y Aldás, 2005, p. 393). Esta técnica permitió reconocer los factores para caracterizar las variables que tuvieron el mayor impacto en atención a las diferentes reformas tributarias en el periodo de estudio considerado (Gráfico 2).

En el eje F1 las variables Ingreso, IVA, P\&G. tienen la mayor correlación (Cuadro 4). Mientras Ocupación, Empleo e IPC tienen la mayor correlación con el eje 2.

La primera componente se le puede denominar componente ventas ya que está relacionada directamente con los Ingresos, el IVA y su reflejo en el P\&G de las empresas. Por tanto, este componente representa cómo las reformas impactaron directamente las ventas por servicios de alojamiento de los hoteles.

\section{GRÁFICO 2}

Componentes principales

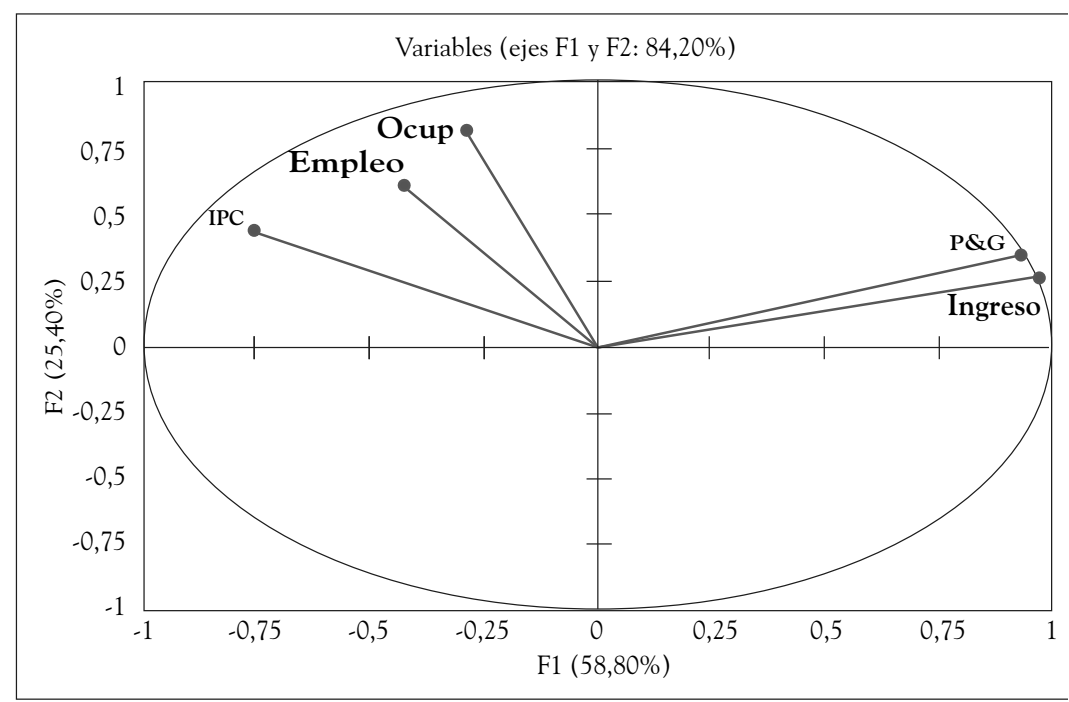

Fuente: Elaboración propia. 


\section{CUADRO 4}

Ejes de los componentes principales

\begin{tabular}{|l|r|r|}
\hline \multicolumn{2}{|c|}{ F1 } & \multicolumn{1}{c|}{ F2 } \\
\hline Ingreso & 26.214 & 4.425 \\
\hline IVA & 25.763 & 4.607 \\
\hline PYG & 24.480 & 8.125 \\
\hline ocup & 2.366 & 45.647 \\
\hline Empleo & 5.038 & 24.646 \\
\hline IPC & 16.138 & 12.550 \\
\hline
\end{tabular}

Fuente: Cálculos del autor.

Al segundo componente se le puede denominar desempeño pues las variables Ocupación y Empleo están más correlacionadas con el segundo eje.

De acuerdo con estas relaciones se determina los años en los que estas variables tuvieron un mayor impacto.

La componente ventas está correlacionada positivamente con los años 2009 a 2014, y a su vez correlacionada negativamente con los años 2004 a 2008 (Gráfico 3). Por su parte la componente desempeño está correlacionada con los primeros años (2004 a 2008) que coincide con los primeros años de promulgadas las reformas. De acuerdo con estos resultados se analiza la evolución del IVA y su impacto en la gestión de este sector hotelero. Como se mencionó y según las pruebas estadisticas realizadas, los cambios en esta tarifa resultan ser un factor determinante y de alta sensibilidad para la gestión y la operación de estos establecimientos.

Los antecedentes de esta tarifa se remontan a la Ley 49 de $1990^{10}$, la cual determinó un impuesto sobre las ventas del $4 \%$ para los hoteles, hostales, residencias y en general para el servicio de hospedaje. La evolución de esta tributación corresponde a la lógica de imposición indirecta a estos servicios, "como muestra clara de la intención de incentivar la adquisición de servicios de hospedaje en el territorio nacional, encontramos que la Ley 488 de 1998 modificó el tratamiento tributario para estos servicios cuando fueran prestados por establecimientos hoteleros o de hospedaje, excluyéndolos de la lista de servicios gravados con IVA". ${ }^{11}$

\footnotetext{
${ }^{10}$ Recuperado de www.corteconstitucional.gov.co/relatoria/1993/C-260-93.htm

${ }^{11}$ Recuperado de http://www.secretariasenado.gov.co/senado/basedoc/ley_0488_1998.html
} 


\section{GRÁFICO 3}

Diagrama de dispersión de las reformas

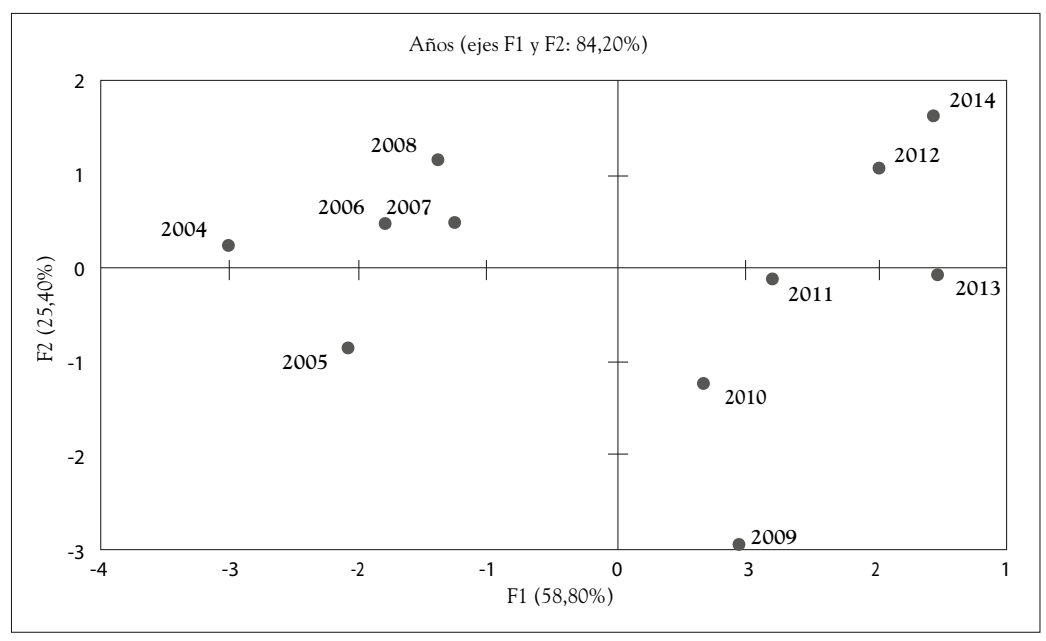

Fuente: Elaboración propia.

Así también, la Ley 633 del 2000, en el marco de la internacionalización del turismo, dio muestras claras de la intención de privilegiar al sector introduciendo una exención del IVA para: “... los servicios turísticos prestados a residentes en el exterior que sean utilizados en territorio colombiano, originados en paquetes vendidos en el exterior y vendidos por agencias operadoras u hoteles inscritos en el Registro Nacional de Turismo ...” ut Infoconsultoria-Yanhaas (2016).

Lo hallado en UT Infoconsultoria-Yanhaas (2016) determinó que en materia de IVA la mayor carga tributaria en el sector alojamiento lo tienen los establecimientos clasificados como "Hoteles", sobre las otras categorías, "encontramos que, en materia de IVA, aunque las sumas de impuestos son mucho más altas, lo cierto es que se sigue encontrando la mayor carga tributaria en el sector de "Alojamiento hoteles", a continuación se ubica el "Alojamiento aparta-hoteles", y "Otros alojamientos". Además de lo anterior, vale la pena resaltar que en materia de IVA este sector de la económica aporta cantidades importantes al fisco nacional" UT Infoconsultoria-Yanhaas (2016).

Explicado este contexto es importante analizar los principales indicadores financieros de estos establecimientos. El impacto en los Ingresos por servicios de 
alojamiento fue analizado a partir de los cambios en el IVA originados por las diferentes reformas tributarias; para tal propósito se ajustó un modelo de regresión múltiple stepwise donde las variables predictivas consideradas fueron: ocupación, servicios ofrecidos por el hotel gravados con IVA, modelo jurídico ${ }^{12}$, utilidades operacionales, total de empleados, tarifa promedio, porcentaje de ocupación de no residentes y porcentaje de IVA gravable.

Los coeficientes de regresión del modelo están estandarizados de manera que se puede comparar las variables con diferente unidad de medida. Los resultados se muestran en el Cuadro 5.

CUADRO 5

Impacto del IVA en ingresos

\begin{tabular}{|c|c|c|c|c|c|c|}
\hline & \multirow{2}{*}{ Modelo* } & \multicolumn{2}{|c|}{ Coeficientes no estandarizados } & \multirow{2}{*}{$\begin{array}{c}\text { Coeficientes } \\
\text { estandarizados }\end{array}$} & \multirow{2}{*}{$t$} & \multirow{2}{*}{ Sig. } \\
\hline & & $\mathrm{B}$ & Error estándar & & & \\
\hline \multirow{9}{*}{1} & (Constante) & $-236,467,857,471$ & $96,553,564,998$ & & $-2,449$ & ,014, \\
\hline & IVA & $-2,185,169,504,496$ & $578,579,167,978$ &,- 011 & $-3,777$ &, 000 \\
\hline & Servicios con IVA & $-91,650,618,286$ & $18,082,265,546$ &,- 016 & $-5,069$ &, 000 \\
\hline & Ocupación & $625,149,095,192$ & $57,707,783,399$ & ,032 & 10,833 &, 000 \\
\hline & Modelo jurídico & $163,989,864,176$ & $23,141,670.097$ & ,023 & 7,086 &, 000 \\
\hline & Utilidades operacionales & 1,561 & ,021 & 634 & 75,529 &, 000 \\
\hline & Empleo & $21,070,394,251$ & $516,347,322$ & ,342 & 40,807 &, 000 \\
\hline & Tarifa & $2,062,182$ & 203,708 & ,031 & 10,123 & ,000 \\
\hline & Ocupación no residentes & $-1,693,549,572$ & $539,407,118$ &,- 010 & $-3,140$ & 002 \\
\hline
\end{tabular}

${ }^{*}$ Variable dependiente: Ingresos.

Fuente: Elaboración propia a partir de muestra desarrollada en UT Infoconsultoria-Yanhaas (2016).

Todas las variables consideradas en el modelo fueron significativas; este es un modelo con un buen ajuste. Las variables utilidad y total de empleados son las que más influyen en la variación de los ingresos por alojamiento, mientras que las variables IVA, servicios ofrecidos por el hotel y gravados con IVA y porcentaje

\footnotetext{
${ }^{12}$ Se consideraron los siguientes modelos jurídicos: Independiente, de Cadena, de Central de compras conjunta, Franquicia y Contratos de cuentas en participación.
} 
de ocupación de no residentes, presentan una relación inversa sobre los ingresos por alojamiento; es decir, se espera que a incrementos en el IVA origine caídas en los ingresos por alojamiento.

Los coeficientes estandarizados estimados se muestran en el Cuadro 6.

CUADRO 6

Coeficientes del modelo IVA en ingresos

\begin{tabular}{|l|c|}
\hline IVA & $-1,10 \%$ \\
\hline Servicios con IVA & $-1,47 \%$ \\
\hline Ocupación & $2,93 \%$ \\
\hline Modelo jurídico & $2,05 \%$ \\
\hline Utilidades operacionales & $57,71 \%$ \\
\hline Empleo & $31,11 \%$ \\
\hline Tarifa & $2,86 \%$ \\
\hline Ocupación no residentes & $-0,88 \%$ \\
\hline
\end{tabular}

Fuente: Elaboración propia a partir de muestra desarrollada en UT Infoconsultoria-Yanhaas (2016).

Se estimó en este modelo que la variable Ingresos tiene una alta dependencia directa con la utilidad operacional y el empleo; mientras que se estimó una dependencia inversa con el IVA, es decir, cada vez que el IVA aumenta un punto porcentual, se espera que los ingresos disminuyan en un 1,1\%.

Este comportamiento inverso entre IVA e ingresos por alojamiento se corroboró en UT Infoconsultoria-Yanhaas (2016, p. 137): "se realizó un análisis con la información institucional reportada por la DIAN en los últimos 15 años y efectivamente se encontró que en el año 2013 (primer año de impacto del cambio de IVA del 10\% al 16\%) los ingresos por venta de habitaciones cayeron o descendieron un 3,57\%".

Demuestra Ut Infoconsultoria-Yanhaas (2016) que, en valores absolutos: "En el año 2013, la caída de la utilidad operacional en el sector hotelero fue de $\$ 9,252$ millones. Esta cifra corresponde en promedio a $\$ 1,542$ millones de utilidad operacional hotelera, por cada punto adicional en que aumentó el IVA ese año". Y concluye: "En el periodo de estudio considerado (últimos 15 años), se estimó que en términos agregados el sector ha dejado de percibir utilidades operacionales por $\$ 40.669$ millones de pesos a precios constantes como consecuencia de los 
cambios en la tasa del IVA aplicada al sector hotelero." uT Infoconsultoria-Yanhaas (2016, p. 138).

Los gestores de estos establecimientos estabilizan los ingresos vía reducción tarifas o volumen de ventas, o en muchos casos a partir de la combinación de estos dos mecanismos. Como era de esperarse esta maniobra es viable para los establecimientos con mayor presencia en los mercados y mayor capacidad financiera. La mediana y la pequeña hotelería no tienen este margen de maniobra.

\section{CONCLUSIONES}

Los últimos gobiernos han propiciado la inversión y la cualificación de la gestión en el sector turismo mediante estímulos tributarios. En especial el sector alojamiento ha tenido un tratamiento diferenciado en el marco de la tributación directa e indirecta. Los estímulos tributarios se fundamentan en una lógica económica basada en que los impuestos que deja de pagar el privado los convierte en infraestructura para generar inversión, consumo intermedio y empleo.

Sin embargo, otra postura considera que si el Estado recaudara estos impuestos que deja de percibir por estímulos fiscales podrían ser invertidos en la infraestructura que requiere el turismo para su desarrollo.

Los estímulos establecidos por la Ley 788 de 2002 cuyo pilar era la exención del impuesto de renta propició un crecimiento inédito de la oferta habitacional en los principales destinos turísticos del país. Por ejemplo, Bogotá durante una década creció a un ritmo promedio de un nuevo hotel por mes.

En este sentido, esta política de estímulos tributarios logró su objetivo de propiciar la inversión en infraestructura habitacional, destinada a servicios de alojamiento. Sin embargo, como la demanda no creció a los mismos ritmos de la oferta, se presentó una sobreoferta habitacional en estos mismos destinos.

Los gestores del sector alojamiento desarrollaron estrategias para mantener su participación en el mercado; muchas se focalizaron en la reducción de tarifas Esto originó una "guerra de tarifas" en la que la mediana y la pequeña hotelería resultaron afectadas. Otras estrategias se limitaron a la reducción del empleo directo como mecanismo para mantener la utilidad operacional. Sin embargo, esta acción afecta directamente la calidad de los servicios.

Estos estímulos atrajeron inversiones especulativas al sector que propiciaron el negocio inmobiliario, antes que hotelero, a través de proyectos como la titula- 
rización. Los inversionistas muy rápido se desalentaron ante la imposibilidad de alcanzar las metas de rendimientos prometidos por los operadores.

Los aumentos en la tarifa del IVA afectan los ingresos de los hoteles; generalmente este ajuste se traslada al usuario de estos servicios. Esta práctica se agudiza con los impuestos no descontables, como el Impuesto Nacional al Consumo (INC), el cual se traslada al consumidor directamente.

En el caso de un impuesto indirecto como el IVA, el cual es pagado por el consumidor final (huésped), tiene un impacto en las utilidades operacionales y los ingresos por alojamiento. Los efectos indirectos son la afectación de las tarifas y el empleo.

Los resultados obtenidos indican una coherencia ente los impactos ocurridos con motivo de las reformas tributarias, los beneficios fiscales y los cambios en la tarifa del IVA. Los aumentos en este impuesto afectan negativamente las tarifas de la hotelería.

Los cambios en el IVA diferencial afectaron directamente los principales indicadores de la gestión y la operación hotelera, como los ingresos y la utilidad. Se pudo determinar la relación inversa entre el IvA y la utilidad operacional; un incremento de un punto en el IVA genera una caída del 1,1\% en la utilidad operacional hotelera.

Se concluye con todo este análisis que los cambios tributarios afectan directamente al sector. Se debe descartar el argumento artificial que cuando se generan ajustes tributarios el impacto para la rentabilidad de la operación hotelera es insustancial.

\section{REFERENCIAS}

CEPAL (2006). Políticas activas para atraer inversión extranjera directa: experiencia internacional y situación de América Latina y el Caribe. Santiago de Chile: Cepal. Debates Contemporáneos sobre el Turismo (2016), Desafios actuales en la Gestión e Innovación del Turismo. Tomo III, Universidad Externado de Colombia.

De la Santa, E (2017), "Fiscal Incentives for Tourism Development in the Philippines", Tourism Planning $\mathcal{E}$ Development.

Friedman, M (1962), Capitalism and Freedom. Chicago: University of Chicago Press. Hair, Anderson, y Tatham, Black (2004), Análisis Multivariante, 5a ed, Upper Sadlle River, NJ: Prentice Hall. 
Johnson, D (1998), Métodos Multivariados Aplicados al Análisis de Datos, Toronto: Thomson Editores.

Leguizamón, M (2017), Métodos y Técnicas de la Medición en Turismo, Bogotá: Universidad Externado de Colombia.

Pérez, C (2004), Técnicas de Análisis Multivariante de Datos, pper Saddle River, NJ: Prentice Hall.

Scheel, A (2010), Evaluación Operacional y Financiera aplicada a la Industria Hotelera, Bogotá: Universidad Externado de Colombia. Bogotá.

Steger, M., y R. Roy (2010), Neoliberalismo: una Breve Introducción, Madrid: Alianza Editorial.

UT Infoconsultoria-Yanhaas (2016), Impacto del Iva y otros Tributos en el Sector Hotelero, Bogotá: Fontur Mincit.

Uriel, E., y J. Aldás (2005), Análisis Multivariado Aplicado, Toronto: Thompson Editores.

\section{Normas citadas}

Congreso de Colombia. (1995). Ley 223 de 1995. Recuperado de http://www. secretariasenado.gov.co/senado/basedoc/ley_0223_1995.html

Congreso de Colombia. (1998). Ley 488 de 1998. Recuperado de http://www. secretariasenado.gov.co/senado/basedoc/ley_0488_1998.html

Congreso de Colombia. (2000). Ley 633 del 2000. Recuperado de http://www. mineducacion.gov.co/1621/articles-270478_recurso_1.pdf

Congreso de Colombia. (2012). Ley 1607 de 2012. Recuperado de http://www. secretariasenado.gov.co/senado/basedoc/ley_1607_2012.html

www.corteconstitucional.gov.co/relatoria/1993/C-260-93.htm

Concepto Dian No. 09115 del 26 de marzo de 2015.

http://www.secretariasenado.gov.co/senado/basedoc/ley_1739_2014.htm

http://www.secretariasenado.gov.co/senado/basedoc/

https://www.dian.gov.co/Prensa/HistoricoCentrales/Ley1819Diciembre2016. pdf

Ley 788 de 2002. Diario oficial No. 45.046, 27 de diciembre de 2002 